\title{
Freight Information Matching Based on Quantum Evolutionary Algorithm
}

\author{
Xiangwei $\mathrm{Mu}^{1, \mathrm{a}^{*}}$, Yan Chen ${ }^{1}$ and Haixia Zhao ${ }^{1}$ \\ ${ }^{1}$ Transportation Management College, Dalian Maritime University, Dalian 116026, China \\ muxiangwei@dlmu.edu.cn
}

Keywords: Freight Information Matching ; Quantum Evolutionary Algorithm ; Genetic Algorithm

\begin{abstract}
In order to improve the efficiency of freight vehicle and cargo matching, a mathematical model was established, which describes target and the related constraints, and quantum evolutionary algorithm was proposed to solve this matching problem. In the experiment, improved quantum evolutionary algorithm was compared with the standard genetic algorithm, and the algorithm parameters were optimized. Experiment results show that quantum evolutionary algorithm has better convergence speed, accuracy and stability, which can efficiently get an optimal freight supply and demand matching plan.
\end{abstract}

\section{Introduction}

In order to solve the vehicle idle and empty problems caused by the asymmetry of vehicle source and cargo source in the cargo business, it emerges a large number of logistics public information platform and distribution sites to promote circulation and sharing of vehicle source and cargo source information.

In recent years, the related research of operating mechanism and technical means about logistics public information platform[1-4]. However, as for the related research of vehicle cargo matching method, which is one of the core functions of a logistics information platform, is just started and not perfect. At present, there are two main methods of vehicle and cargo matching method. The first method, in view of the low query efficiency and poor experience for user caused by low degree of logistics information standardization, starts from information semantic retrieval, builds a semantic knowledge model of vehicle source and supply of cargo, and implements more complex supply and demand relationship between semantic retrieval and recommendations through semantic reasoning ability[5,6]. Nevertheless, because of the complicated knowledge semantic modeling process and reasoning process, this method is difficult to be realized in practical application.

The second method establishes evaluation index system for vehicle cargo information resources. In addition, using correlation method, it conducts evaluation and comprehensive ranking for the matching degree of vehicle source and cargo source according to historical information[7-8]. This method requires a mass of business data related to index system to evaluate, which causes a certain impact for user experience. And the generated matching scheme of this method is influenced by the subjective evaluation factors. Further more, this method mainly aims at each car source or cargo source in evaluation and matching without considering unified resource assignment problem, and causes easily the vicious circle of "excellent resources is always recommended, new resources is difficult to find out", that is to say, the "zero start" problem of new resources.

In this paper, a mathematical model was set up to solve the problem of supply and demand matching of vehicle and cargo, the objective function and constraints are described mathematically, and the quantum evolutionary algorithm with constraint penalty was designed to match the vehicle and cargo information. The method contributes to finding the optimized matching plan for logistics public information platform in a large number of vehicle source and cargo source information, which can improve the efficiency of freight distribution from the whole point of view. 


\section{Vehicle and cargo matching model}

the VCM problem can be described as follows: the chief demand of vehicle and cargo matching problem is to recommend automatically appropriate cargo source and transport vehicles to the vehicle owner and cargo owner according to the known information of vehicle source and cargo source, in order to make the vehicle owner and cargo owner conduct business connection after their choice. Under the premise of meeting certain business agreement, Make full use of the existing information resources, find the best matching scheme to meet a certain business objectives in different matching schemes, and achieve real-time matching and recommended service for vehicles and goods[10-12].

In order to make full use of the information of vehicle source and cargo source, the matchingprocess needs to consider the pursuit of maximizing the matching rate of vehicle cargo $R$, and consider comprehensively total cost $\mathrm{C}$ caused by all recommended program after matching should be minimum. Cost function $\mathrm{C}$ can be considered for the comprehensive composition of such variables : distance to the vehicle current position and the cargo shipment location, user time window 、 cargo destination distance and so on. The objective function is defined as follows:

$$
\max Z=w_{1} R+w_{2}(C+1)^{-1}
$$

The cost function $\mathrm{C}$ is simplified as the distance between current position of vehicle and cargo departure position, based on the above model parameters, model assumptions and objective function analysis. In practical application, cost function can be constructed other forms according to the specific needs. Vehicle and cargo matching model is as follows:

$$
\begin{array}{ll}
\max & Z=w_{1} R+\frac{w_{2}}{\sum_{i=1}^{I} \sum_{k=1}^{K} l_{i k} x_{k i}+1} \\
\text { s.t. } \quad \sum_{i=1}^{I} x_{k i} \leq M_{I}, M_{I} \leq I, \forall k \\
\quad \sum_{k=1}^{K} x_{k i} \leq M_{K}, M_{k} \leq K, \forall i \\
\quad \sum_{i=1}^{I} d_{i} x_{k i} \leq g b_{k}, \forall k, g \geq 1 \\
d_{i} x_{k i} \leq b_{k}, \forall i, \forall k \\
\quad i=1,2, \ldots, I \\
k=1,2, \ldots, K
\end{array}
$$

Which $K(k=1,2,3 \ldots . K)$ vehicles and $I(i=1,2, \ldots, I)$ cargo transport demand need to matching, and the transport capacity of each vehicle is known as $b_{k}$, each cargo demand is $d_{i}, l_{k i}$ is the distance between the current location of vehicle and the location of cargo. The distance matrix of all vehicles and cargo demand points is $L$, a matching $x_{k i}$ for vehicle and cargo demand is defined as

$$
x_{k i}=\left\{\begin{array}{l}
1, \text { matching of vehicle } \mathrm{k} \text { and cargo i } \\
0, \text { other }
\end{array}\right.
$$

Definition 1 A Vehicle-Cargo Matrix, a VCM matrix consisting of all matching $x_{k i}$, is a solution to the matching problem of vehicle and cargo.

$$
V C M=\left(\begin{array}{ccc}
x_{11} & \cdots & x_{1 I} \\
\vdots & \ddots & \vdots \\
x_{K 1} & \cdots & x_{K I}
\end{array}\right)
$$

Definition 2 The formula for calculating the VCM matching rate $\mathrm{R}$ is 


$$
R=\frac{\sum_{i=1}^{I} \sum_{k=1}^{K} x_{k i}}{K \times I}
$$

Constraint formula (3) limits that the transport demand for each shipment has at most $M_{I}$ matching vehicles; Constraint formula (4) limits that each vehicle can match $M_{K}$ cargo transport requirements at best; Constraint formula (5) requires that the overall cargo weight of each vehicle that matches all the cargo transport needs is less than $g b_{k}$, which makes vehicles with strong transport capacity can get more matching program; Constraint formula (6) requires that the weight of the goods in each plan recommended by each vehicle is less than the weight of the transport vehicle, avoiding giving the vehicle an overweight cargo.

\section{Matching method based on quantum evolutionary algorithm}

3.1 Quantum evolutionary algorithm. QEA(Quantum-inspired Evolutionary Algorithm), based on the quantum state vector representation, which makes the probability amplitude representation of a quantum bit act on chromosome's encoding and makes an individual of each chromosome express superposition of multiple states at the same time[13]. In quantum computation, physical media as information storage unit is a two-state quantum system, called quantum bit, and referred to as qubit. qubit's difference with classical bit is that it can be in the superposition state of two quantum states.

$$
|\psi>=\alpha| 0>+\beta \mid 1>
$$

Thereinto, $\alpha, \beta$ are plural, and respectively express the probability amplitude of $\mid 0>$ and $\mid 1>$.they satisfy

$$
|\alpha|^{2}+|\beta|^{2}=1
$$

Quantum individuals represented by quantum bits can be used to enrich the diversity of the population, better global searching ability, and avoid the local optimal solution. The evolution process of QEA uses the quantum rotation gate $U(\theta)$ to change the probability amplitude of the quantum bits, so that the quantum individual is gradually approaching the optimal solution. Quantum rotation gate calculation formula is:

$$
\left[\begin{array}{l}
\alpha^{\prime} \\
\beta^{\prime}
\end{array}\right]=U(\theta)\left[\begin{array}{l}
\alpha \\
\beta
\end{array}\right]=\left[\begin{array}{cc}
\cos (\theta) & -\sin (\theta) \\
\sin (\theta) & \cos (\theta)
\end{array}\right]\left[\begin{array}{l}
\alpha \\
\beta
\end{array}\right]
$$

Thereinto, $\left[\alpha^{\prime}, \beta^{\prime}\right]^{\mathrm{T}}$ is a new quantum bit probability amplitude after the evolution of the quantum rotation gate. $\theta$ is the rotation angle and satisfies the following formula:

$$
\theta=S(\alpha, \beta) \Delta \theta
$$

$S(\alpha, \beta)$ and $\Delta \theta$ were used to determine the direction of rotation and the angle increment.

3.2 Algorithm design. The bit length of the quantum individual $\mathrm{Q}$ is $K \times I$. The $\mathrm{n}$-th bit $\alpha_{n}$ is given randomly in the initial state, and gives the corresponding position $\beta_{n}$ according to the formula (9). It defines the measured value of the Q's n-th quantum bit is:

$$
Q_{n}^{\psi}=\left\{\begin{array}{l}
1, t h_{n} \geq \alpha_{n}{ }^{2} \\
0, t h_{n}<\alpha_{n}^{2}
\end{array}\right.
$$

Thereinto, $t h_{n}$ is the threshold randomly given for each bit after each quantum evolution, and satisfies $0<t h_{n}<1$.Thus, the greater the probability that the $\mathrm{n}$ quantum bit of quantum individual is 1 , the smaller the $\alpha_{n}$. The quantum individual measurement value is composed of the measured value of each quantum bit.

$$
Q^{\psi}=\left\{Q_{1}^{\psi}, Q_{2}^{\psi}, \ldots, Q_{K \times I}^{\psi}\right\}
$$

The decoding process needs to map the measured values of each quantum individual's bits to each element of the VCM matrix. Mapping relation is:

$$
x_{k i}=Q_{(k-1) \times I+i}^{\psi}
$$


According to the objective function formula (1) of vehicle matching model, the fitness function of the quantum individual can be defined as:

$$
\begin{aligned}
& F\left(Q^{\psi}\right)=\frac{w_{1} \sum_{n=1}^{K \times l} Q_{n}^{\psi}}{K \times I}+\frac{w_{2}}{1+\sum_{n=1}^{K \times l} Q_{n}^{\psi} L(n)} \\
& L(n)=l_{\left\ulcorner\frac{n}{I}\right\rceil, n-l\left(\left\lceil\frac{n}{I}-1-1\right)\right.}
\end{aligned}
$$

In order to confirm $S(\alpha, \beta)$ and $\Delta \theta_{n}$, we should establish selection policy table. As shown in Table 1.

Table1 rotation angle selection strategy

\begin{tabular}{|c|c|c|c|c|c|c|}
\hline \multirow{2}{*}{$Q_{n}^{\prime}$} & \multirow{2}{*}{ Best $Q_{n}^{\prime \prime}$} & \multirow{2}{*}{$\Delta \theta_{n}$} & \multicolumn{5}{|c|}{$S\left(\alpha_{n}, \beta_{n}\right)$} \\
\cline { 4 - 7 } & & & $\alpha_{n} \beta_{n}>0$ & $\alpha_{n} \beta_{n}<0$ & $\alpha_{n}=0$ & $\beta_{n}=0$ \\
\hline 0 & 0 & $\delta$ & -1 & +1 & +1 & 0 \\
\hline 0 & 1 & $\delta$ & +1 & -1 & 0 & 1 \\
\hline 1 & 0 & $\delta$ & -1 & +1 & 1 & 0 \\
\hline 1 & 1 & $\delta$ & +1 & -1 & 0 & +1 \\
\hline
\end{tabular}

Thereinto, $\delta$ is the angle increment of each adjustment $\Delta \theta$ which can be adjusted by static or dynamic methods. The strategy of adjustment compares $Q_{n}{ }^{\psi}$ (every bit measurement value of quantum individual) with Best $Q_{n}{ }^{\psi}$ (bit measurement value corresponding to the historical optimal quantum individual). The rotation angle $\theta_{n}$ is obtained by the way of looking up table and the formula.

\section{Experimental}

The experimental running environment is $2.5 \mathrm{GHz} \mathrm{CPU}$, memory $4 \mathrm{~GB}$, using JDK1.6 to programme. At present, In view of the experimental data vehicle cargo matching problem, there is not a standard test database. In order to use traversal algorithm to obtain exact solutions in feasible time and compared with the QEA algorithm optimal solution. This paper limits the size of the experimental data. The experimental data includes 5 vehicles and 6 cargo transport requirements, as shown in Table 2. Thereinto, $b_{k}$ column is the transport capacity of each vehicle (ton), $d_{i}$ row is each kind of cargo transport demand quantity (ton), and others express the point of cargo and the distance of the vehicle (kilometer). Set the relevant parameters of the vehicle cargo matching problem model, respectively be : $\mathrm{K}=5 ; \mathrm{I}=6 ; w_{1}=0.6 ; w_{2}=0.4 ; M_{I=} M_{K}=4 ; g=2, \mathrm{~L} 、 b_{k}$ and $d_{i}$. As shown in Table 2.this paper uses traversal method to obtain the exact solutions of the above data, and all vehicle cargo matching schemes are 1073741824 kind $\left(2^{5^{*} 6}=1073741824\right)$, the traversal algorithm analyzes and calculates about 50000 scheme per second about six hours, and gets the exact fitness solution 0.283226 .

Table2 Experimental data

\begin{tabular}{|c|c|c|c|c|c|c|c|}
\hline & $\mathbf{c 1}$ & $\mathbf{c 2}$ & $\mathbf{c 3}$ & $\mathbf{c 4}$ & $\mathbf{c 5}$ & $\mathbf{c 6}$ & $\boldsymbol{b}_{\boldsymbol{k}}$ \\
\hline $\mathbf{v 1}$ & 2 & 10 & 5 & 6 & 9 & 12 & 100 \\
\hline $\mathbf{v 2}$ & 3 & 4 & 1 & 2 & 5 & 7 & 100 \\
\hline $\mathbf{v 3}$ & 20 & 30 & 15 & 23 & 32 & 40 & 90 \\
\hline $\mathbf{v 4}$ & 16 & 9 & 20 & 11 & 5 & 17 & 90 \\
\hline $\mathbf{v 5}$ & 7 & 6 & 9 & 10 & 15 & 3 & 60 \\
\hline $\boldsymbol{d}_{\boldsymbol{i}}$ & 60 & 70 & 80 & 70 & 50 & 50 & -- \\
\hline
\end{tabular}

The QEA algorithm step is shown as follow:

step1. quantum group initialization : When the quantum population is initialized, it needs to give relevant parameters of vehicle cargo matching model: K、I、L、 $b_{k}, d_{i}, w_{1}, w_{2} 、 M_{I}, M_{k}$ 、 $g$, give quantum individual number $\mathrm{P}$ and the length of bit position $(\mathrm{K} \times \mathrm{I})$. The initial measurement 
threshold $\left(t h_{n}\right)$ and the bit position quantum state of each individual quantum $(\alpha=\beta=\sqrt{0.5})$ are given randomly.

step2. quantum individual fitness calculation : According to the formula (13), it can decide the initial measurement value $Q^{\psi}$ of every quantum individual. Testing the quantum bit measurement value is 1 , if it is not in accordance with constraint formula (6), set the value of the bit to 0 . At the same time, the bit quantum state is set to: $\alpha=1, \beta=0$, thus, the solution is at least a weak feasible solution for all the quantum individual measurement values.

step3. selection of optimal quantum individuals : If there is a strongly feasible solution for the current quantum group, namely, $\xi=0$, the fitness of the quantum individual with the strong feasible solution is compared with that of the historical optimal quantum individual $\left(B e s t Q^{\psi}\right)$, and keeps the optimal strong feasible solution quantum individual Best $Q^{\psi}$. If there is no strong feasible solution, then the optimal weak feasible solution quantum individual is saved as Best $Q^{\psi}$.

step4. algorithm exit condition judgment : The premise of algorithm exit is that historical optimal quantum individual $\left(\right.$ Best $\left.Q^{\psi}\right)$ is strongly feasible solution, and also it should meet one of the following conditions: $F\left(\right.$ Best $\left.Q^{\psi}\right)$ is greater than a given threshold value, or the times. of quantum group evolution is larger than a given threshold value. Algorithm exit decision success is to enter the step6, otherwise the algorithm to step5.

step5. quantum group evolution : Quantum rotation gate angle increment $(\Delta \theta)$ is given. According to the rotation strategy set in Table 1 and formula (11) (12), the bit quantum state of each quantum individual is improved, and a new generation of quantum group is generated. Algorithm goes to step2.

step6. optimal quantum individual decoding : According to the formula (15), the measured value of the historical optimal quantum individual is resolved to SVM, and the result is output.

In Experimental, parameters of quantum evolution algorithm are: Quantum group size is 100 quantum individuals; evolution times are $1000 ; \Delta \theta=0.01 \pi$. When the algorithm is used for about 0.6 seconds, the optimal strong feasible solution is 0.28320 , and the optimal solution is obtained in the 358th evolution for the first time. The evolution process is shown in Table 3

Table3 Quantum Evolution Process

\begin{tabular}{|c|c|}
\hline $\begin{array}{c}\text { Evolution } \\
\text { times }\end{array}$ & $\begin{array}{c}\text { Optimal } \\
\text { fitness }\end{array}$ \\
\hline 57 & 0.282649 \\
\hline 69 & 0.282685 \\
\hline 73 & 0.282703 \\
\hline 88 & 0.282721 \\
\hline 111 & 0.28274 \\
\hline 248 & 0.282759 \\
\hline 330 & 0.28292 \\
\hline 358 & 0.2832 \\
\hline
\end{tabular}

Using the same vehicle cargo matching model, parameters and data, QEA algorithm and GA (Genetic Algorithm) are compared, assume the number of the population is 100, and run randomly and independently 30 times. Algorithm results statistics as shown in Table 4.

Table4 Comparison of QEA and GA algorithms

\begin{tabular}{|c|c|c|c|}
\hline $\begin{array}{c}\text { algorith } \\
\mathrm{m}\end{array}$ & $\begin{array}{c}\text { average } \\
\text { error } \\
(\mathrm{E}-\end{array}$ & $\begin{array}{c}\text { standard } \\
\text { deviation } \\
(\mathrm{E}-\end{array}$ & $\begin{array}{c}\text { evolution speed } \\
\text { of reaching } \\
\text { optimal } \\
05)\end{array}$ \\
\hline GA & 24.2 & 16.01 & 463 \\
\hline QEA & 3.3 & 10.81 & 191 \\
\hline
\end{tabular}


Statistical results shows that the QEA algorithm has better performance than the genetic algorithm GA.

(1) Algorithm convergence speed. QEA algorithm can get the optimal solution of the current population through evolution of average 191 times, however, the GA algorithm needs to evolve 463 times on average, which shows that the QEA algorithm can get the optimal solution with less iteration times, and the convergence speed is faster.

(2) Algorithm accuracy. Compared with the exact solution, the average error of QEA's 30 independent running is 3.3E-05, the average error of GA algorithm is 24.2E-05, QEA average error reduced $86 \%$ than GA, indicating that the QEA has better accuracy and better global search ability.

(3) Algorithm stability. The standard deviation of QEA algorithm 30 times running results is $10.81 \mathrm{E}-05$, and GA algorithm is 16.02E-05, indicating that the result values of QEA algorithm's 30 times running are more concentrated and stable.

\section{Acknowledgement}

This research is funded by China Postdoctoral Science Foundation (2014M551063), Scientific Research Fund of Liaoning Provincial Education Department(L2014203), Liaoning Social Science Planning Fund (L14BGL012) and the Fundamental Research Funds for the Central Universities(3132016046).

\section{References}

[1] J. Namjoshi, A. Gupte. 2009 IEEE International Conference on Cloud Computing. Bangalore, 147, (2009)

[2] Li Yongping. Research on the hanging logistics information platform construction based on the Internet of things technology[D]. ChangAn University,2014.

[3] Dong Qianli. Journal of Traffic and Transportation Engineering. 4,58(2002)

[4] Li Li, Zhou Xiaorong, Manufacturing automation,9,88(2013).

[5] Gu Jiajing. Vehicle cargo matching system based on Semantic Web Technology [D]. Tsinghua University, 2013.

[6] J. E. Cuadrado, C. Preist, S. Williams. Artificial Intelligence: Methodology, Systems, and Applications. 96(2004)

[7] Li Hui. Research of the loading vehicle cargo supply and demand matching on logistics information service platform [D]. Beijing Jiaotong University, 2015.

[8] Sun Chengzhi, Yang Yi,Wu Jianbin, Shi Mengmeng, Gao Lan, Li Lindiao. Logistics technology and Application, 04,130(2014).

[9] E.E. Zachariadis, C.D. Tarantilis, C.T. Kiranoudis. Expert Systems with applications, 36,1070(2009).

[10]Zhai Yong,Liu Jiehua,Zhang Wei,Fan Mingqu. Journal of transportation engineering and information, $\mathbf{0 3}, 91(2008)$.

[11]P.P. Repoussis, C.D. Tarantilis, Ioannou G. Journal of the Operational Research Society, 58, 355(2007)

[12]F. Errico, G.Desaulniers, M. Gendreau, et al. European Journal of Operational Research, 249, 55(2016).

[13]Zhou Zengwei, Xu Tao, Gong Ming, Li Chuanfeng, Hu Yong, Yang Yong, Guo Guangcan. Progress in Physics, 02,127(2009). 